\title{
The transmission of Leishmania infantum chagasi by the bite of the Lutzomyia longipalpis to two different vertebrates
}

\author{
Nagila FC Secundino ${ }^{*}$ Vanessa C de Freitas, Carolina C Monteiro, Ana-Clara AM Pires, Bruna A David and \\ Paulo FP Pimenta
}

\begin{abstract}
Background: Sandflies are vectors of Leishmania, the causative agent of leishmaniasis in mammalian hosts, including humans. The protozoan parasite is transmitted by the sandfly bite during salivation that occurs at the moment of blood feeding. The components of vector saliva include anticlotting and vasodilatory factors that facilitate blood flow and immunomodulatory factors that inhibit wound healing and quell the immune response. Not surprisingly, these factors also play important roles in the establishment of Leishmania infection. To date, the majority of knowledge that has been generated regarding the process of Leishmania infection, including $L$. infantum chagasi transmission has been gathered by using intradermal or subcutaneous inoculation of purified parasites.

Findings: This study presents the establishment of a transmission model of Leishmania infantum chagasi by the bite of Lutzomyia longipalpis, the vector of American visceral leishmaniasis. The parasites were successfully transmitted by infected sandfly bites to mice and hamsters, indicating that both animals are good experimental models. The L. infantum chagasi dose that was transmitted in each single bite ranged from 10 to 10, 000 parasites, but $75 \%$ of the sandflies transmitted less than 300 parasites.

Conclusions: The strategy for initiating infection by sandfly bite of experimental animals facilitates future investigations into the complex and dynamic mechanisms of visceral leishmaniasis. It is important to elucidate the transmission mechanism of vector bites. This model represents a useful tool to study L. infantum chagasi infection transmitted by the vector.
\end{abstract}

Keywords: transmission, bite, leishmaniasis, Lutzomyia longipalpis

\section{Findings}

Despite the fact that Leishmania is transmitted exclusively by sandfly vectors, a reproducible animal model of Leishmania infection transmitted by sandfly bite was only described in the year 2000 [1].

Early investigations showed that infected $P$. papatasi sandflies can release from 0 to over 1,000 L. major promastigotes through their proboscides by forced feeding [2]. However, a transmission model by bite with $P$. duboscqui infected with $L$. major found that the parasite

\footnotetext{
* Correspondence: nagila@cpqrr.fiocruz.br

Laboratory of Medical Entomology of the Centro de Pesquisas René Rachou, Fundação Oswaldo Cruz - MG. Av. Augusto Lima, 1715 Barro Preto, Belo Horizonte, 30190-002, Minas Gerais, Brazil
}

numbers inoculated in the host skin by one insect vector alone could vary from 10 to 100,000 [3].

Considering the New World species of Leishmania, using a feeding device of chick skin membrane over culture medium showed that L. longipalpis sandflies infected with Leishmania mexicana (an unnatural vector/parasite pair) expelled an average of 1,000 parasites per fly [4]. Thus, even though L. longipalpis is the vector of L. infantum chagasi in nature, it is permissive in the laboratory to infection with other Leishmania species.

In consideration of the continuing prevalence of American visceral leishmaniasis and the paucity of related studies in the literature, our goal was to develop an L. infantum chagasi transmission model by bite,
C Biomed Central

(ㄷ) 2012 Secundino et al; licensee BioMed Central Ltd. This is an Open Access article distributed under the terms of the Creative Commons Attribution License (http://creativecommons.org/licenses/by/2.0), which permits unrestricted use, distribution, and reproduction in any medium, provided the original work is properly cited. 
using its proven vector, L. longipalpis. In addition, we aimed to define the amount of parasites that are directly expelled into the mammalian host, which has never before been carried out.

To evaluate the sandfly infection, colonized two- to four-day-old L. longipalpis females (Lapinha Cave strain) were infected by feeding through a chick skin membrane (feeding device) on mouse blood containing $4 \times 10^{6} / \mathrm{mL}$ L. infantum chagasi (MHOM/BR/70/BH46) promastigotes. After two, six, nine and fourteen days, the midguts of infected flies were dissected and the parasite load was estimated by hemocytometer counting.

For Transmission by bite, fourteen-day infected flies were transferred to small plastic vials (3-dram volume, $4.8-\mathrm{cm}$ height, $1.8-\mathrm{cm}$ diameter) covered at one end with a $0.25-\mathrm{mm}$ nylon mesh. Balb/C mice and hamsters were anesthetized by Thiopental injection. Clamps were used to hold the mesh end of each vial flat against the animals' ears so that the fly had access to the ear skin for feeding over a period of 1-2 $\mathrm{h}$ in the dark. The animals were then euthanized and the exposed ears were dissected for testing the parasite presence. One infected sandfly was used for each of the transmission experiments (Figure 1). All animals were maintained at the Animal Care Facility of the FIOCRUZ-MG under specific pathogen-free conditions and were used in accordance to a study protocol approved by the FIOCRUZ Ethical Committee for Animal Use (CEUA; license number LW30/10).

Exposed-bite animal ears were processed for DNA extraction and real-time PCR as described elsewhere [3]. Parasite quantification was performed by automatic comparison with the specific set of standard samples prepared in parallel to each set of test samples. The number of L. infantum chagasi in each sample was summarized as the mean of the two median values from the three reactions in each run. Twenty-eight or 30 ears from each group (mouse or hamster) were processed.

A total of 640 L. longipalpis females from 800 individuals ingested the infected bloodmeal containing $L$. infantum chagasi promastigotes. A group of 300 sandflies from this collection were dissected and evaluated up to 14 days after infection. Seventy-two hours after the infective bloodmeal, $100 \%$ of the sandflies were positive for viable promastigotes, with an average of 14,000 promastigotes per midgut. At six days post-infection, there was an approximate $70 \%$ reduction in parasite growth and survival ( $p=0.0009)$, with an infection average of 3, 000 parasites per sandfly; this change may reflect loss of parasites due to fly defecation or the hostile digestion process, as described previously [5]. At 9 to 14 days post-infection, the observed L. infantum

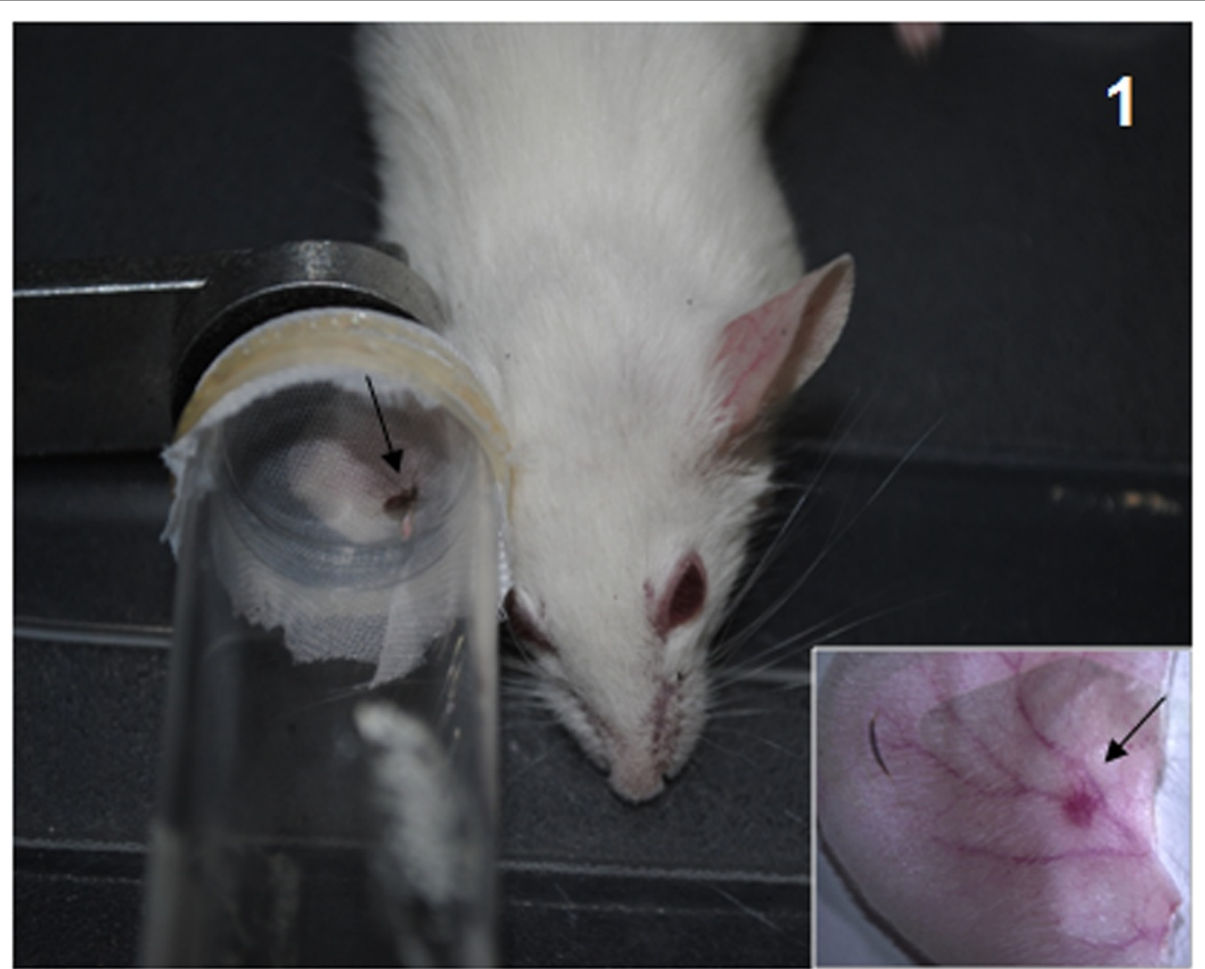

Figure 1 L. longipalpis sandfly biting a Balb/C mouse. A single sandfly was confined within a vial and allowed to bite the animal's entire ear. Note the blood engorgement of the sandfly (arrow). Inset: Dissected mouse ear showing the bite site as a small red dot (arrow). 


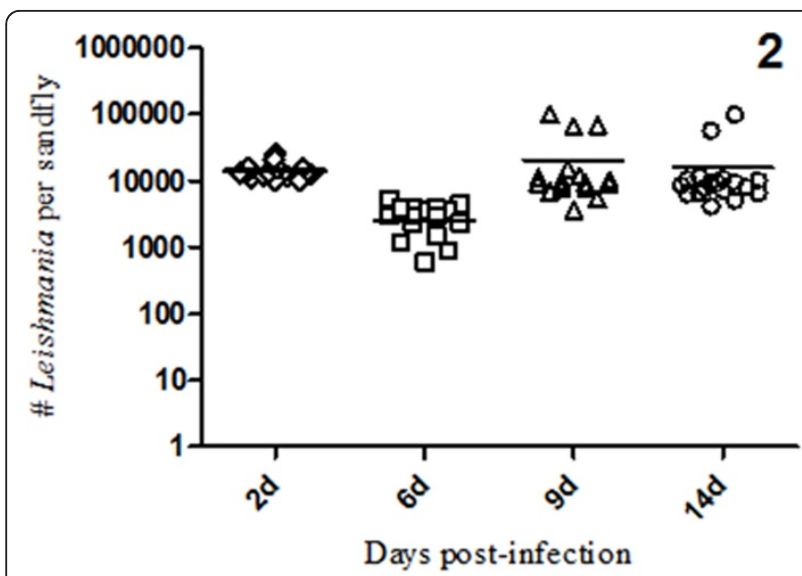

Figure 2 Parasite numbers in infected L. longipalpis ( 2 to 14 days after the infective bloodmeal). Flies were infected by membrane feeding on mouse blood containing 4 million logarithmic phase promastigotes $/ \mathrm{mL}$. Midguts were dissected on the indicated days post-feeding and scored for numbers of viable parasites.

chagasi growth inside the sandfly indicated that a mature infection had been established reaching an average of 10,000 promastigotes/midgut (Figure 2).

To develop a transmission model for laboratory use, we initially used two groups of animals: mice and hamsters. Twenty-eight ears of each animal group were exposed to a single infected $L$. longipalpis. The detection and quantification of L. infantum chagasi transmitted by the sandfly bites were carried out using real-time PCR. The parasite doses expelled from the sandflies to the mouse ears ranged from 4 to 15,000 (mean: 1,255), and to hamster ears ranged from 8 to 10,000 (mean: 1,122). There was no significant difference in the parasite number expelled by L. longipalpis bites into mice or hamsters, suggesting each can be used as model for transmission of $L$. infantum chagasi by its infected sandfly vector (Figure 3 ).

When only a single sandfly was allowed to bite the mouse ears $(\mathrm{n}=30$ ears), real-time PCR detected the presence of $L$. infantum chagasi in 27 ears. The three that were negative for PCR detection of parasites also did not show outward signs of bites. In the infected ears, the parasite quantification showed that transmitted dose by the bite of a single L. longipalpis ranged from 10 to 10, 000 (mean: 1, 002). Finally, it was determined that $75 \%$ of the sandflies transmitted $<300 \mathrm{~L}$. infantum chagasi in each ear (Figure 4).

In the New World, particularly in Brazil, leishmaniasis has followed the massive migration of people from rural areas into urbanized regions. In addition, the density of metropolitan areas and ease of travel between the two areas has led to increasing incidence rates of this vector-transmitted infectious disease [6]. Studies with the

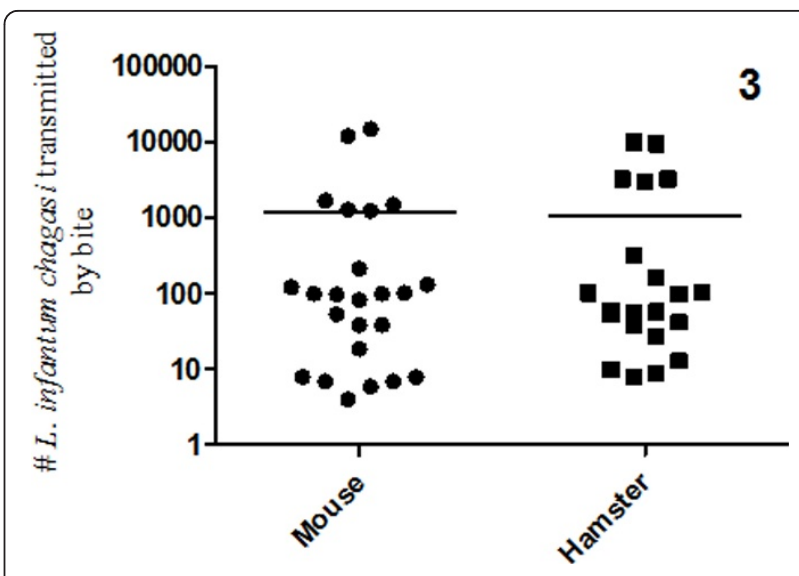

Figure 3 L. infantum chagasi transmitted to the mouse and hamster ears. The parasite numbers were obtained by real-time PCR of dissected ears at two hours after the bites. No significant differences were observed for parasite transmission by the various sandflies.

Brazilian vector species are, thus, necessary to understand the underlying mechanisms of parasites, vector, and the vertebrate hosts, as well as the complex interactions between the three, which support disease onset and persistence.

In order to establish, for the first time, a transmission model of laboratory infection of visceral leishmaniasis, we used L. longipalpis infected with L. infantum chagasi to initiate infection by bite in laboratory animals. This approach probably is superior to the traditional needle inoculation method, which eliminates the natural

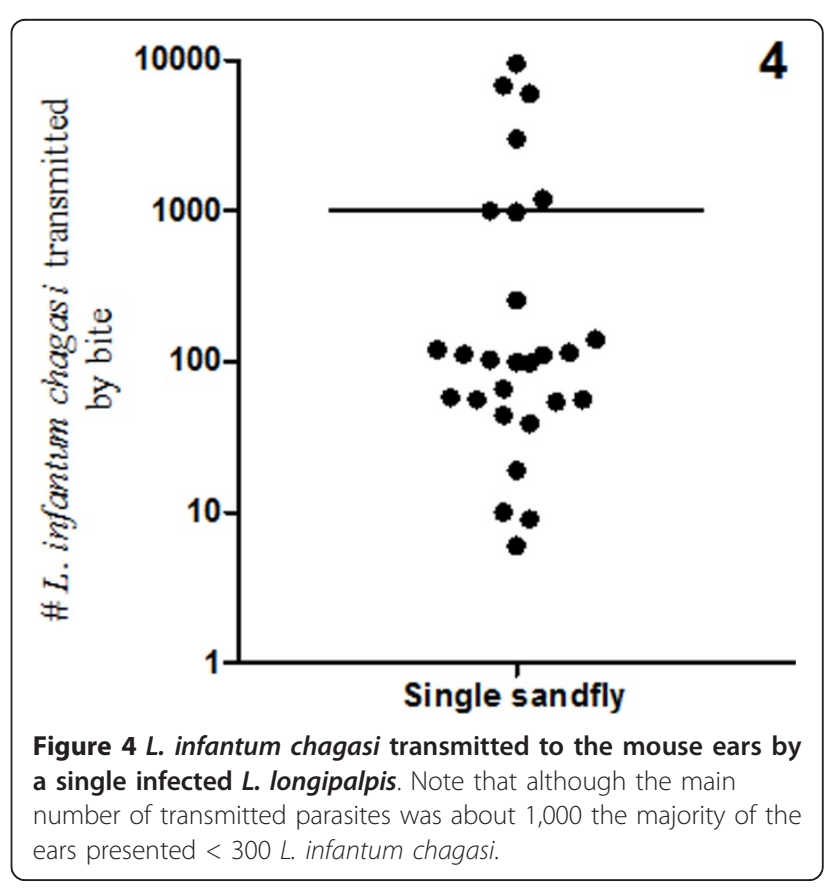


transmission features (saliva, natural doses expelled through the proboscides).

Initially, we evaluated and compared hamsters and Balb/C mice as mammalian hosts for experimental infection. We concluded that there is no significant difference between these two animals in their utility as a model for transmission of visceral leishmaniasis by the vector bite. The two animals were bitten equally and the parasite numbers expelled into the ears by the L. longipalpis sandfly were similar, demonstrating that they are effective mammalian hosts for transmission of laboratorial infection.

Our results showed that the amount of $L$. infantum chagasi promastigotes delivered by a single L. longipalpis to the ears ranged from 4 to 10, 000 (mean: 1, 002). Coincidently, these numbers are similar to the findings from the force-feeding study [2] and others, in which $L$. longipalpis were artificially infected with $L$. mexicana and then forced to expel parasites into culture medium [4].

Interestingly, it is worth noting that in an experimental study of Old World cutaneous leishmaniasis the amount of $L$. major injected into mouse skin by a single sandfly, $P$. duboscqui could reach 100,000 parasites, but the majority of sandflies transmitted $\sim 600$ parasites [3].

Collectively, with our analysis, these findings indicated that the New World sandfly L. longipalpis cannot transmit as many parasites as the Old World sandfly. The majority of parasite loads transmitted by $L$. longipalpis are very low, since $75 \%$ of them transmitted $<300$ parasites in the laboratory conditions. Only a few flies transmitted around 10, 000 parasites, a number that is still 10 -fold less than that of the P. duboscqui. Further studies using other vector sandfly species are necessary to better understand this difference.

This study provided a strategy for initiating infection of experimental animals by vector bite that will facilitate future investigations into the complex and dynamic mechanisms of visceral leishmaniasis. The use of this model of parasite transmission may help to develop new strategies for prevention and treatment of leishmaniasis, a human life-treating disease.

\section{Acknowledgements}

This work was supported by grants from Fundação de Apoio a Pesquisa do Estado de Minas Gerais (FAPEMIG), Conselho Nacional de Desenvolvimento Tecnológico (CNPq), and Fundação Oswaldo Cruz (FIOCRUZ). We would also like to thank the FAPEMIG for its financial support of Vanessa C. de Freitas through a Junior Post-Doctoral Fellowship (CBB 00260/09).

\section{Authors' contributions}

NFCS conceived and designed the experiments; NFCS and PFPP analyzed the data and wrote the paper; VCF, CCM, ACAMP, BAD performed the experiments. All authors read and approved the final version of the manuscript.

\section{Competing interests}

The authors declare that they have no competing interests.

Received: 29 November 2011 Accepted: 19 January 2012

Published: 19 January 2012

\section{References}

1. Kamhawi K, Belkaid Y, Modi G, Rowton E, Sacks D: Protection against cutaneous leishmaniasis resulting from bites of uninfected sandflies. Science 2000, 290:1351-1354.

2. Warburg A, Schlein S: The effect of post-bloodmeal nutrition of Phlebotomus papatasi on the transmission of Leishmania major. Am J Trop med Hyg 35:926-930.

3. Kimblin N, Peters N, Debrabant A, Secundino N, Egen J, Lawyer P, Fay M, Kamhawi S, Sacks D: Quantification of the infectious dose of Leishmania major transmitted to the skin by single sandflies. PNAS 2008, 105:10125-10130.

4. Rogers ME, Ilg T, Nikolaev AV, Ferguson MA, Bates PA: Transmission of cutaneous leishmaniasis by sandflies is enhanced by regurgitation of fPPG. Nature 2001, 430:463-467.

5. Secundino N, Kimblin N, Peters N, Lawyer P, Capul A, Beverley S, Salvatore JT, Sacks D: Proteophosphoglycan confers resistance of Leishmania major to midgut digestive enzymes induced by blood feeding in vector sandflies. Cellular Microbiology 2010, 12:906-918.

6. Ashford RW: The leishmaniasis as emerging and reemerging zoonoses. Int J Parasitol 2000, 30:1269-81.

doi:10.1186/1756-3305-5-20

Cite this article as: Secundino et al:: The transmission of Leishmania infantum chagasi by the bite of the Lutzomyia longipalpis to two different vertebrates. Parasites \& Vectors 2012 5:20.

\section{Submit your next manuscript to BioMed Central and take full advantage of:}

- Convenient online submission

- Thorough peer review

- No space constraints or color figure charges

- Immediate publication on acceptance

- Inclusion in PubMed, CAS, Scopus and Google Scholar

- Research which is freely available for redistribution 